\title{
Efficacy of XELOX plus Bevacizumab in Brain Metastasis from Rectal Cancer
}

\author{
Yoichiro Yoshida Seiichiro Hoshino Naoya Aisu Masayasu Naito \\ Syu Tanimura Takamitsu Sasaki Shinsuke Takeno Yuichi Yamashita \\ Department of Gastroenterological Surgery, Fukuoka University Faculty of Medicine, \\ Fukuoka, Japan
}

\section{Key Words}

Colorectal cancer · Chemotherapy · Brain metastasis · Bevacizumab · XELOX

\begin{abstract}
Brain metastasis $(B M)$ is rare in colorectal cancer (CRC) patients. Although BM from CRC is a late-stage phenomenon with an extremely poor prognosis, some subsets of patients would benefit from a multidisciplinary management strategy. The prognosis of patients with BM from CRC was associated with the curability of the therapy for BM and the number of metastatic organs. Metastatic brain tumors are generally treated with radiotherapy because many anticancer drugs cannot cross the blood-brain barrier. Here, we present a case treated with XELOX (capecitabine and oxaliplatin) plus bevacizumab for BM from rectal cancer. To our knowledge, this is the first report of a patient who was successfully treated for BM from CRC without radiotherapy. The findings could lead to a paradigm shift in the use of chemotherapy for BM from CRC.

(c) 2014 S. Karger AG, Basel
\end{abstract}

\section{Introduction}

Brain metastasis (BM) is rare in colorectal cancer (CRC) patients. In a recent cohort study, BM was diagnosed in $8.5 \%$ of patients. The cumulative incidence of BM after 5 years was estimated at $16.3 \%$ in patients with lung cancer, $9.8 \%$ in patients with renal cell carcinoma, $7.4 \%$ in patients with melanoma, and $5.0 \%$ in patients with breast cancer [1]. Previous studies have reported the incidence of BM in CRC to range from 1 to $3 \%[1,2]$. Although BM from CRC is a late-stage phenomenon with an extremely poor prognosis, some subsets of patients would benefit from a multidisciplinary management strategy. The 
Yoshida et al.: Efficacy of XELOX plus Bevacizumab in Brain Metastasis from Rectal Cancer

prognosis of patients with BM from CRC was associated with the curability of the therapy for $\mathrm{BM}$ and the number of metastatic organs [3].

Bevacizumab (BV) is effective in the treatment of metastatic CRC (mCRC). However, it is unclear whether BV is safe and effective in the treatment of BM from CRC. BV recently gained accelerated approval from the US Food and Drug Administration for progressive primary brain tumors, with a low rate (approx. 3\%) of intratumoral hemorrhage [4, 5]. More recent studies showed that BV is safe in patients with BM [6-9]. Recently, the efficacy of BV for patients with breast cancer and BM was reported [10]. We present the case of a patient treated with XELOX + BV for BM from rectal cancer.

\section{Case Report}

A 62-year-old man was referred to our hospital with abdominal fullness and gait disturbance. Barium enema revealed a nearly obstructing tumor in the rectum. Stenosis prevented the endoscope from passing through. The patient needed a fasting because of stenosis of the rectum. Abdominal computed tomography (CT) revealed more than 10 hepatic metastases. Head CT and MRI showed two cerebellar metastases (fig. 1). The patient underwent a resection of BM and loop transverse colostomy. We started XELOX + BV [BV $(7.5 \mathrm{mg} / \mathrm{kg})$ and oxaliplatin $\left(130 \mathrm{mg} / \mathrm{m}^{2}\right)$ on day 1 plus capecitabine $\left(1,000 \mathrm{mg} / \mathrm{m}^{2}\right)$ twice daily on days 1-14, every 3 weeks] which was administered on postoperative day 28 [11]. After 4 cycles of XELOX + BV, CT and MRI showed a reduction in tumor size (fig. 2). Laboratory studies revealed a serum carcinoembryonic antigen (CEA) level of 3,460 ng/ml, which improved to $936 \mathrm{ng} / \mathrm{ml}$ after 4 cycles of chemotherapy. Observed adverse events that had appeared before were only a peripheral neuropathy in the acute stage (grade 1) and hand-foot syndrome (grade 1). Hematological toxicity, surgical site infection and incisional hernia were not noted.

After 8 months, tumor progression has not occurred, and the patient is still undergoing therapy with XELOX + BV.

\section{Discussion}

Patients with BM from CRC have a poor prognosis because they often have substantial extracranial metastatic disease. Traditionally, the therapeutic goal in many of these patients has been to palliate debilitating neurologic symptoms because most of these patients die of systemic disease. However, new advances in mCRC management - including the incorporation of monoclonal antibody therapies with BV [12], cetuximab [13], and panitumumab [14] - are enhancing the outcomes of patients with systemic disease. Although these targeted therapies have improved management of systemic disease, they are not as effective for BM given the restrictions on delivery into a tumor caused by the blood-tumor and blood-brain barriers (BBB). In light of these advances, the management of BM from CRC may deserve reconsideration.

It is clear that careful patient selection influences the relative success of brain lesion resection. In a retrospective review from the late 1990s, Wronski and Arbit [15] evaluated the disease-specific outcomes of surgical resection in 73 patients with mCRC to the brain who met specific criteria for surgery. The patients were evaluated for the extent of disease. Those with limited extracranial disease and a life expectancy in excess of 6 months were considered as well as those with a maximum of 2-3 accessible lesions. 
The mechanism of $\mathrm{BV}$ in brain parenchymal disease is still unknown; whether it is a result of direct interaction on the tumor vasculature and/or the BBB itself is unclear. Most drugs are not able to enter the central nervous system because of the BBB. This restriction particularly affects drugs that are not substrates for active transport into the central nervous system, and high molecular weight therapeutic modalities, such as monoclonal antibodies, antisense oligonucleotides, viral vectors and stem cells [16]. However, some studies have shown that VEGF may provide new opportunities for manipulating the permeability of the BBB $[17,18]$. Furthermore, previous studies in glioma models have demonstrated a fine balance between VEGF and angiopoietin-2, a proapoptotic factor in angiogenesis [19]. It has been described that the BBB is abnormal with tumors $>0.5 \mathrm{~mm}$, and might affect the integrity of astrocytes and the endothelial cells of the BBB [20]. Larger tumors result in an increased risk of ischemia, further disrupting the BBB [21]. More studies are in progress to evaluate the role of $\mathrm{BV}$ with chemotherapy in previously treated BM originating from lung cancer and also in reducing side effects of the central nervous system after radiotherapy in patients with primary brain cancer, melanoma, and head and neck cancer $[22,23]$. In our case, XELOX + BV suppressed BM. Therefore, there is a possibility that BV might cross the $\mathrm{BBB}$ and penetrate brain tumors in sufficient concentrations to synergize with anticancer drugs.

\section{Disclosure Statement}

The authors have no conflicts of interest to disclose.

\section{References}

1 Schouten LJ, Rutten J, Huveneers HA, Twijnstra A: Incidence of brain metastases in a cohort of patients with carcinoma of the breast, colon, kidney, and lung and melanoma. Cancer 2002;94:2698-2705.

-2 Sundermeyer ML, Meropol NH, Rogatko A, et al: Changing patterns of bone and brain metastases in patients with colorectal cancer. Clin Colorectal Cancer 2005;5:108-113.

-3 Noura S, Ohue M, Shingai T, Fujiwara A, Imada S, Sueda T, Yamada T, Fujiwara Y, Ohigashi H, Yano M, Ishikawa 0: Brain metastasis from colorectal cancer: prognostic factors and survival. J Surg Oncol 2012;106:144-148.

4 Friedman HS, Prados MD, Wen PY, et al: Bevacizumab alone and in combination with irinotecan in recurrent glioblastoma. J Clin Oncol 2009;27:4733-4740.

5 Agha CA, Ibrahim S, Hassan A, Elias DA, Fathallah-Shaykh HM: Bevacizumab is active as a single agent against recurrent malignant gliomas. Anticancer Res 2010;30:609-611.

-6 Socinski MA, Langer CJ, Huang JE, et al: Safety of bevacizumab in patients with non-small-cell lung cancer and brain metastases. J Clin Oncol 2009;27:5255-5261.

7 Besse B, Lasserre SF, Compton P, Huang J, Augustus S, Rohr UP: Bevacizumab safety in patients with central nervous system metastases. Clin Cancer Res 2010;16:269-278.

8 Labidi SI, Bachelot T, Ray-Coquard I, et al: Bevacizumab and paclitaxel for breast cancer patients with central nervous system metastases: a case series. Clin Breast Cancer 2009;9:118-121.

$\$ 9$ Bhaskara A, Eng C: Bevacizumab in the treatment of a patient with metastatic colorectal carcinoma with brain metastases. Clin Colorectal Cancer 2008;7:65-68.

$\checkmark 10$ Yamamoto D, Iwase S, Tsubota Y, Sueoka N, Yamamoto C, Kitamura K, Odagiri H, Nagumo Y: Bevacizumab in the treatment of five patients with breast cancer and brain metastases: Japan Breast Cancer Research Network-07 trial. Onco Targets Ther 2012;5:185-189.

-11 Yoshida Y, Hoshino S, Aisu N, Naito M, Miyake T, Tanimura S, Yamashita Y: Pilot study of the early start of chemotherapy after resection of primary colorectal cancer with distant metastases (Pearl Star 01). World J Surg Oncol 2013;11:39.

12 Kabbinavar FF, Hambleton J, Mass RD, Hurwitz HI, Bergsland E, Sarkar S: Combined analysis of efficacy: the addition of bevacizumab to fluorouracil/leucovorin improves survival for patients with metastatic colorectal cancer. J Clin Oncol 2005;23:3706-3712. 
13 Cunningham D, Humblet Y, Siena S, Khayat D, Bleiberg H, Santoro A, Bets D, Mueser M, Harstrick A, Verslype C, Chau I, Van Cutsem E: Cetuximab monotherapy and cetuximab plus irinotecan in irinotecan-refractory metastatic colorectal cancer. N Engl J Med 2004;351:337-345.

-14 Van Cutsem E, Peeters M, Siena S, Humblet Y, Hendlisz A, Neyns B, Canon JL, Van Laethem JL, Maurel J, Richardson G, Wolf M, Amado RG: Open-label phase III trial of panitumumab plus best supportive care compared with best supportive care alone in patients with chemotherapy-refractory metastatic colorectal cancer. J Clin Oncol 2007;25:1658-1664.

15 Wronski M, Arbit E: Resection of brain metastases from colorectal carcinoma in 73 patients. Cancer 1999;85:1677-1685.

-16 De Boer AG, Gaillard PJ: Strategies to improve drug delivery across the blood-brain barrier. Clin Pharmacokinet 2007;46:553-576.

17 Ferrara N, Gerber HP, LeCouter J: The biology of VEGF and its receptors. Nat Med 2003;9:669-676.

18 Ay I, Francis JW, Brown RH: VEGF increases blood-brain barrier permeability to Evans blue dye and tetanus toxin fragment C but not adeno-associated virus in ALS mice. Brain Res 2008;1234:198-205.

19 Zadeh G, Guha A: Molecular regulators of angiogenesis in the developing nervous system and adult brain tumors. Int J Oncol 2003;23:557-565.

20 Gerstner ER, Fine RL: Increased permeability of the blood-brain barrier to chemotherapy in metastatic brain tumors: establishing a treatment paradigm. J Clin Oncol 2007;25:2306-2312.

-21 Dietrich WD, Busto R, Halley M: The importance of brain temperature in alterations of the blood-brain barrier following cerebral ischemia. J Neuropathol Exp Neurol 1990;49:486-497.

22 ClinicalTrials.gov: Bevacizumab in reducing CNS side effects in patients who have undergone radiation therapy to the brain for primary brain tumor, meningioma, or head and neck cancer (accessed August 27, 2012).

23 ClinicalTrials.gov: A study of bevacizumab in combination with first- or second-line therapy in subjects with brain metastases due to non-squamous NSCLC (PASSPORT) (accessed August 27, 2012).
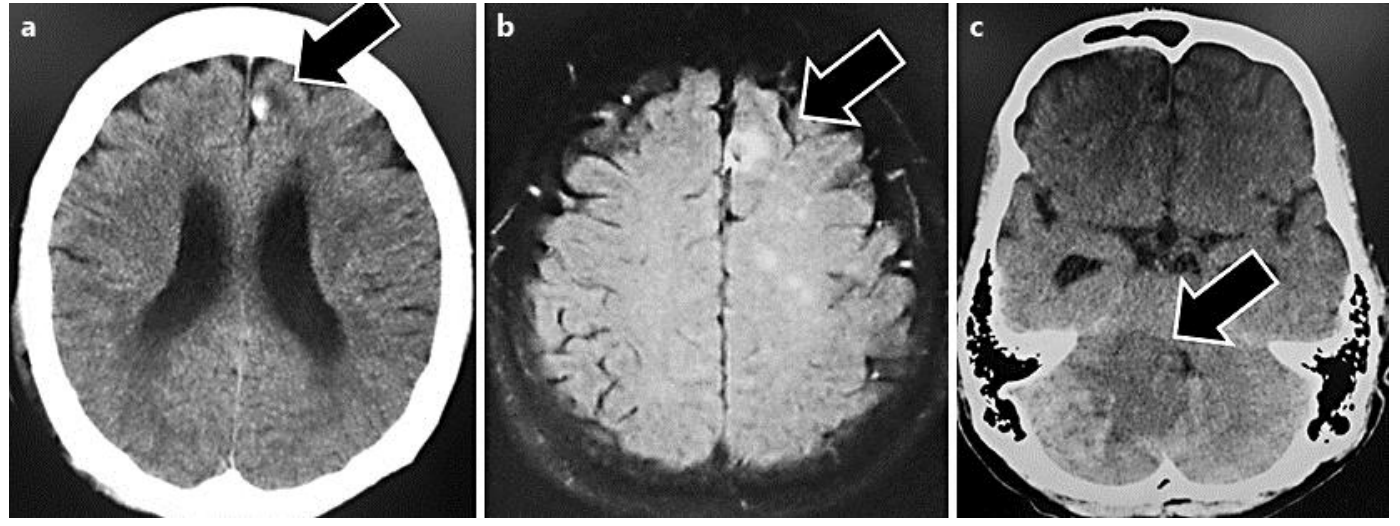

Fig. 1. a Head CT and b MRI showing a BM. c Resection of a BM. 


\section{Case Reports in Oncology}

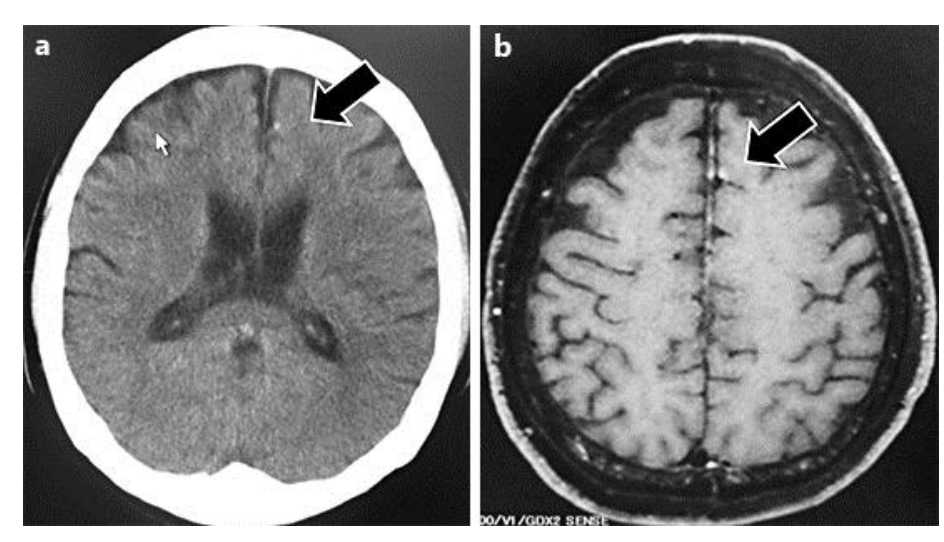

Fig. 2. a Posttreatment brain CT and b MRI of the metastatic tumor showing a partial response.

\begin{tabular}{l|l}
\hline DOI: $10.1159 / 000360132$ & $\begin{array}{l}\text { @ 2014 S. Karger AG, Basel } \\
\text { www.karger.com/cro }\end{array}$ \\
\hline $\begin{array}{l}\text { Yoshida et al.: Efficacy of XELOX plus Bevacizumab in Brain Metastasis from Rectal } \\
\text { Cancer }\end{array}$
\end{tabular}

